\title{
Magnetization Transfer and Amide Proton Transfer MRI of Neonatal Brain Development
}

\author{
Yang Zheng, ${ }^{1}$ Xiaoming Wang, ${ }^{1}$ and Xuna Zhao ${ }^{2,3}$ \\ ${ }^{1}$ Department of Radiology, Shengjing Hospital of China Medical University, No. 36, Sanhao Street, Heping District, \\ Shenyang 110004, China \\ ${ }^{2}$ Division of MR Research, Department of Radiology, Johns Hopkins University, Baltimore, MD, USA \\ ${ }^{3}$ Philips Healthcare, Beijing, China
}

Correspondence should be addressed to Xiaoming Wang; wangxm024@163.com

Received 3 August 2016; Accepted 10 October 2016

Academic Editor: Haifeng Wang

Copyright (C) 2016 Yang Zheng et al. This is an open access article distributed under the Creative Commons Attribution License, which permits unrestricted use, distribution, and reproduction in any medium, provided the original work is properly cited.

Purpose. This study aims to evaluate the process of brain development in neonates using combined amide proton transfer (APT) imaging and conventional magnetization transfer (MT) imaging. Materials and Methods. Case data were reviewed for all patients hospitalized in our institution's neonatal ward. Patients underwent APT and MT imaging (a single protocol) immediately following the routine MR examination. Single-slice APT/MT axial imaging was performed at the level of the basal ganglia. APT and MT ratio (MTR) measurements were performed in multiple brain regions of interest (ROIs). Data was statistically analyzed in order to assess for significant differences between the different regions of the brain or correlation with patient gestational age. Results. A total of 38 neonates were included in the study, with ages ranging from 27 to 41 weeks' corrected gestational age. There were statistically significant differences in both APT and MTR measurements between the frontal lobes, basal ganglia, and occipital lobes (APT: frontal lobe versus occipital lobe $P=0.031$ and other groups $P=0.00$; MTR: frontal lobe versus occipital lobe $P=0.034$ and other groups $P=0.00$ ). Furthermore, APT and MTR in above brain regions exhibited positive linear correlations with patient gestational age. Conclusions. APT/MT imaging can provide valuable information about the process of the neonatal brain development at the molecular level.

\section{Introduction}

Brain development is a gradual process of continuous maturation, with varying degrees of brain maturity associated with each different developmental period. Maturation of the neonatal and infant brain is a rapid process, occurring predominantly from five months gestational age to approximately one year after birth. During this period, maturation of the developing brain primarily involves neuronal myelination, accomplished via oligodendroglial cell proliferation. This neuroglial cell proliferation is characterized by the synthesis of various proteins, leading to increased total protein content of developing brain tissue.

However, the process of myelination is not homogeneous, generally progressing from caudal to rostral, central to peripheral, and dorsal to ventral [1-5]. Consequently, the different regions of the brain exhibit concurrent differences at the molecular level across each stage of development [6]. Concordantly, the regional progression of myelination produces corresponding differences in appearance upon imaging of the developing brain, exhibiting a consistent pattern of changes on conventional magnetic resonance imaging (MRI) sequences over the course of maturation.

With the development and wide availability of MR, the diffusion tensor imaging (DTI) is used to evaluate the neonatal brain development more frequently, especially in showing the structure of white matter fiber with obvious advantages. DTI can quantify and display the diffusion properties of water molecule in brain tissues, and it is usually used to display white matter microstructure $[7,8]$. Magnetic resonance spectroscopy (MRS) is also applied for evaluation of brain development. In ${ }^{1} \mathrm{H}$-MRS, the changes of $\mathrm{N}$-acetylaspartate 
(NAA), Creatine (Cr), Choline (Cho), myo-Inositol (mI), glutamate (Glu), glutamine (Gln), Glu + Gln (Glx), and so forth in the neonatal period with gestational age/region can be observed $[9,10] .{ }^{31} \mathrm{P}-\mathrm{MRS}$ can evaluate brain development from the perspective of energy metabolism with changes of phosphomonoesters (PME), phosphodiesters (PDE), phosphocreatine (PCr), and so forth [11]. DTI and MRS can evaluate the brain development process from the point of white matter microstructure and cerebral metabolic substance, while some proteins, cholesterol, and lipids are also associated with the brain development. Magnetization transfer (MT) and amide proton transfer (APT) imaging are sensitive to semisolid macromolecules (cholesterol and some lipids) and proteins, and so they can help to detect the changes of the above substances during neonatal brain development period at molecular level.

MT is an MRI technique that offers the ability to quantify structural differences in the central nervous system (CNS). MT imaging contrast is achieved through interactions between protons bound to semisolid macromolecules and the free water protons of biological tissue [12]. MT-MRI utilizes a radiofrequency $(\mathrm{RF})$ pulse applied only to the protons of the semisolid macromolecules, which become saturated. The tightly bound protons in the macromolecular pool then undergo transfer of saturation to water, which modulates MR signal. The effects of MT in tissue can be quantified by calculating the MT ratio (MTR), which indicates the percentage of full or partial MR signals generated by saturation of the biological macromolecules. Predictably, the molecular differences between various tissues yield correspondingly divergent MTR values. In the brain [13], the primary semisolid macromolecular determinants of measured MTR values include cholesterol and other lipids.

APT imaging, a newer technique derived from MT imaging, accomplishes molecular MR imaging based on the principles of CEST $[14,15]$. APT imaging generates tissue contrast through the in vivo detection and quantification of endogenous free proteins and polypeptide chains in tissues. Relatively higher APT signals generally indicate elevated exchange rates resulting from increased protein concentration [16, 17]. Although the clinical applications of APT imaging remain in their investigative stages, this modality has demonstrated promise for the evaluation of brain development [18] and the characterization and grading of brain tumors [19-23].

Thus, the purpose of this study was to investigate the relationship between APT and MT signal and gestational age during neonatal brain development in order to further elucidate the complex mechanisms of maturation during this period of brain development.

\section{Materials and Methods}

2.1. Patient Population. Case data were reviewed for all patients hospitalized in our institution's neonatal ward between December 2013 and June 2014. The patient population of this study were hospitalized for the reasons of respiratory tract infections, fever, skin infections, and diarrhea.
Clinically, these reasons may result in brain lesions. When clinicians suspect that there may be brain lesion, a head MR examination should be ordered. We screened the neonates without nervous system disease for further study. Exclusion criteria included history of brain abnormality established prenatally, birth asphyxia, congenital malformations of the brain, mental retardation, and other diseases of the central nervous system. No intravenous contrast was administered for any portion of the MRI examinations. This study was approved by the local Ethical Committee (ethical approval code: 2013PS280K). We obtained informed consent from the patients' guardians and permission from their primary clinicians based on clinical status before additional APT/MT imaging. Conventional MRI examination is followed by APT/ MT imaging where sedation has to be used only once for the same patient. The sedative is chloral hydrate with high security for newborns.

2.2. Conventional MRI Examination. Patients underwent sedation prior to MRI using a $5 \%$ chloral hydrate $(50 \mathrm{mg} / \mathrm{kg})$ enema administered by an anesthesiologist 30 minutes prior to the study and were monitored by the clinically responsible physician throughout the examination. All examinations were performed on a Philips 3.0 Tesla (3 T) MRI system with pencil beam (pencil beam is a kind of $B_{0}$ shimming method through a pencil beam volume shimming algorithm) and second-order shimming (Achieva 3T TX; Philips Healthcare Systems, Best, Netherlands), using a body coil for transmission and an eight-channel sensitivity-encoding (SENSE) receiver coil. Each examination was interpreted separately by two experienced radiologists.

The conventional brain MRI examination included T1WI, T2WI, and DWI sequences. A fast-field echo (FFE) sequence was performed for T1WI using the following parameters: TR of $200 \mathrm{~ms}$; TE of $2.3 \mathrm{~ms}$; FOV of $180 \times 161 \mathrm{~mm}^{2}$; matrix of $224 \times 162$; slice thickness of $5 \mathrm{~mm}$. Parameters for the turbo spin-echo (TSE) sequence used for T2WI were as follows: TR of $4.6 \mathrm{~ms}$; TE of $200 \mathrm{~ms}$; FOV of $180 \times 155 \mathrm{~mm}^{2}$; matrix of $224 \times 162$; slice thickness of $5 \mathrm{~mm}$. Parameters for the spinecho (SE) sequence used for DWI were as follows: TR of $2500 \mathrm{~ms}$; TE of shortest time; FOV of $200 \times 200 \mathrm{~mm}^{2}$; matrix of $124 \times 124$; slice thickness of $5 \mathrm{~mm}$.

\subsection{APT- and MT-MRI Examination}

2.3.1. APT/MT Image Acquisition. We used a single protocol that could be processed to generate both APT and MT images simultaneously. We used the raw data to calculate both APT and MTR values. The listed parameters apply to both imaging techniques.

Axial T1WI was used for positioning of the neonates at the level of the basal ganglia prior to image acquisition. The APT/MT imaging protocol was tailored to minimize local magnetic field $\left(\mathrm{B}_{0}\right)$ inhomogeneity and optimize signal noise ratio (SNR) while maintaining an acceptable duration of scanning time for clinical applications. The protocol selected 


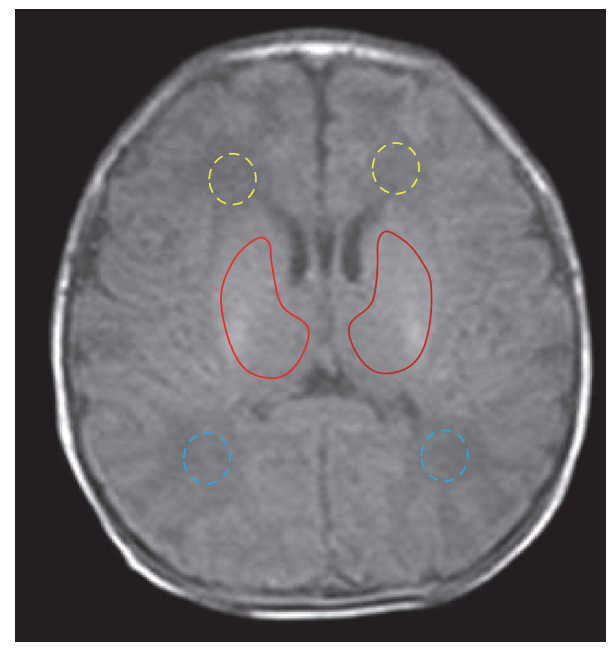

(a)

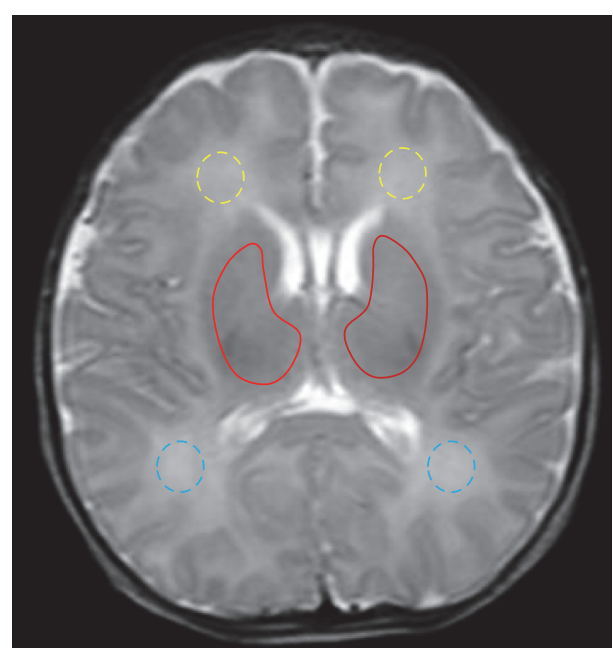

(b)

FIGURE 1: ROIs selection. Images from T1WI (a) and T2WI (b) sequences in the conventional MRI examination are referenced for the selection of ROIs in this study. For all neonates, ROIs are chosen bilaterally in the frontal lobe deep white matter (yellow dotted line), basal ganglia (solid red), and occipital lobe white matter (blue dotted line) bilaterally.

for APT/MT imaging employed an RF saturation time of $500 \mathrm{~ms}$ [24] (the maximum permitted by the body coil used for the examinations).

TSE with a turbo factor (TF) of 38 was used for singleslice acquisition. A multiacquisition method with multiple RF pulses was performed to enhance SNR for MT and APT and included eight acquisitions at \pm 3.5 ppm offset from water frequency [25]. Image acquisition utilized the following parameters: TR of $4000 \mathrm{~ms}$; TE of $8.1 \mathrm{~ms}$; matrix of $108 \times 71$; FOV of $170 \times 145 \mathrm{~mm}$; slice thickness of $5 \mathrm{~mm}$; SENSE factor of 2 , and scan time of $4 \mathrm{~min}, 16 \mathrm{~s}$.

As mentioned above, APT/MT imaging included multiple acquisitions with multiple RF pulses. Over the course of the acquisition process, the images were obtained using different frequency offsets from water. The specific selected frequency offsets are as follows (parentheses indicate multiple acquisitions and the number performed): $0, \pm 0.25, \pm 0.5$, $\pm 0.75, \pm 1, \pm 1.5, \pm 2, \pm 2.5, \pm 3$ (2), \pm 3.25 (4), \pm 3.5 (8), \pm 3.75 (4), $\pm 4(2), \pm 4.5, \pm 5, \pm 6 \mathrm{ppm}$, and $15.6 \mathrm{ppm}$. An unsaturated image was used to normalize the signal.

2.3.2. APT/MT Postprocessing and Data Analysis. For the ATP analysis, raw data from the image acquisition was imported to an interactive data language program (IDL; Research Systems, Inc., Boulder, CO, USA) used for data analyses and reconstruction of pseudo-color images. This software was used to calculate a voxel-based $\mathrm{Z}$ spectrum. A 12 th-order polynomial was then employed to fit the entire $\mathrm{Z}$ spectrum and identify the point of lowest intensity on the $\mathrm{Z}$ spectrum. This information was used to characterize the inhomogeneity of the $\mathrm{B}_{0}$ field and subsequently to obtain field correction of the $\mathrm{Z}$ spectrum. The corrected $\mathrm{Z}$ spectrum data were applied to the MTR asymmetry (MTRasym) analysis using symmetrical \pm 3.5 ppm offset data points. Finally, APT images were generated from the MTRasym values that were calculated at the selected offsets using the following equation: $\operatorname{MTRasym}(3.5 \mathrm{ppm})=S_{\text {sat }} / S_{0}(-3.5 \mathrm{ppm})-S_{\text {sat }} / S_{0}(3.5 \mathrm{ppm})$, where $S_{\text {sat }} / S_{0}$ represents the ratio of signals obtained with $\left(S_{\text {sat }}\right)$ and without $\left(S_{0}\right)$ saturation. The measured APT values were used to reflect the relative magnitude of APT-weighted effects on generated images.

With the same technique and similar method, the MTR was defined according to the equation: $\mathrm{MTR}=1-S_{\text {sat }} / S_{0}$. The measured MT spectra (plotted as a function of saturation frequency offset, relative to water) were corrected for $\mathrm{B}_{0}$ field heterogeneity effects on a pixel-by-pixel basis. Conventional MTR images were calculated from the saturated images at a selected offset of $15.6 \mathrm{ppm}$ [25].

2.4. Selection of ROIs and Image Analysis. Following automated analysis of the raw acquisition data, APT and MT images generated by the software were comparatively analyzed by both senior diagnostic radiologists. With T1WI and T2WI images used as references, the process of image analysis began with the selection of ROIs. For all neonates in the study population, the ROIs included deep white matter in both frontal lobes, bilateral basal ganglia, and deep white matter in both occipital lobes (Figure 1). For ROIs selection, attempts were made to exclude the skull and cerebrospinal fluid (including the cerebral ventricles) in order to avoid associated signal interference.

Each ROI was carefully defined using the drawing function on the clinical workstation; the APT and MT values measured within the ROIs were recorded. The mapping process was performed three times for each ROI to yield the average APT/MT values. The magnitude of APT/MT values measured in the regions of acquisition was used to reflect the relative signal intensity of the ROIs on APT and MT images, with higher values manifesting increased signal intensity. 
2.5. Statistical Analysis. Statistical analyses were performed using SPSS for Windows (Version 17.0, Chicago, IL). Quantitative data were reported as mean \pm standard deviation $(\bar{X} \pm S) . P<0.05$ was interpreted as statistically significant.

Independent two-sample $t$-test was employed to assess for significant differences in APT/MT values measured between ROIs on the left and right side at the same level of the brain. In the absence of significant differences between measurements in each hemisphere, values for each side were averaged by region and recorded for further analysis. APT and MTR values measured in each region (frontal lobe, basal ganglia, and occipital lobe) were analyzed for correlation with gestational age (in days) via Pearson's correlation analysis. ANOVA was applied to assess for statistically significant differences between APT and MTR values individually measured in frontal lobe deep white matter, basal ganglia, and occipital lobe deep white matter.

\section{Results}

3.1. Patient Population. A total of 38 neonates without brain abnormalities were included in the study. The patient population included both preterm and full-term infants, with corrected gestational age ranging from 27 to 41 weeks and median gestational age of 36 weeks \pm 4 days.

3.2. APT and MT by Hemisphere and Brain Region. For all the neonates, both APT and MTR showed significant differences between the regions. The measured APT and MTR values were highest in the basal ganglia, followed by the occipital lobe and lowest in the frontal lobe (Tables 1 and 2): APT: frontal lobe white matter mean $\pm \mathrm{SD}=0.70 \pm 0.29$, basal ganglia mean $\pm \mathrm{SD}=1.30 \pm 0.31$, and occipital lobe white matter mean $\pm \mathrm{SD}=0.86 \pm 0.32$ and frontal lobe versus occipital lobe $P=0.031$, frontal lobe versus basal ganglia $P=$ 0.00 , and basal ganglia versus occipital lobe $P=0.00$. MTR represents frontal lobe white matter mean $\pm \mathrm{SD}=12.09 \pm 1.28$, basal ganglia mean $\pm \mathrm{SD}=18.16 \pm 2.34$, and occipital lobe white matter mean $\pm \mathrm{SD}=12.90 \pm 1.09$ and frontal lobe versus occipital lobe $P=0.034$, frontal lobe versus basal ganglia $P=0.00$, and basal ganglia versus occipital lobe $P=0.00$.

3.3. APT/MT and Gestational Age. APT values measured at all three regions exhibited a linear, positive correlation with gestational age (Figure 2). The strengths of correlation (reported as correlation coefficients) between APT values and gestational age observed in the three regions were as follows (in descending order): occipital lobe white matter $(r=0.87)$, frontal lobe white matter $(r=0.85)$, and basal ganglia $(r=$ $0.80)$.

We observed the MTR measurements (Table 2) in all three regions exhibited a linear, positive correlation with gestational age (Figure 3). Correlation coefficients observed in the three regions were as follows (in descending order): occipital lobe white matter (0.66), frontal lobe white matter (0.46), and basal ganglia (0.46).
Figure 4 shows the T1WI, T2WI, APT, and MTR images of neonates with different gestational ages and shows the changes in MR during brain development.

\section{Discussion}

Initially proposed by Wolff and Balaban in 1989 [26], MTMRI has since earned frequent application for the evaluation of brain and muscular tissue. Many prior studies have demonstrated the efficacy of MT-MRI in reflecting both the density and extent of myelination in the CNS [27-32]. APT, a newer MRI technique based on MT, generates contrast through the exchange between amide protons and water protons in order to reflect the protein content of tissues. The structural and molecular information generated by these MRI techniques offers promise in the study of the brain development. The purpose of this study was to examine the changes in different parts of the neonatal brain with increasing gestational age using MT and APT imaging.

Normally the components of the brain's internal environment and its physical and chemical properties are vital to the survival of brain cells and brain development [33, 34]. However, the neonatal brain constitutes a dynamic environment with continuous substance exchange within its internal environment. The process of neonatal brain development manifests as neuroglial cell proliferation and myelination. Neuroglial cell proliferation is observed as an increase in cell density accompanied by the synthesis of proteins for myelination $[2,3,35,36]$. Neuroglial cell proliferation both precedes and contributes to myelination, providing both essential proteins and cytoplasmic granules containing myelin lipid precursors. Consequently, the process of myelination is associated with continuously increasing protein content in the brain during the course of development and maturation until stabilizing in adulthood.

The progressive and vigorous increase in cell density and protein content during neonatal brain maturation produces highly variable water content and complex biochemical changes in the developing brain tissue, in stark contrast to the comparative stability of adult brain tissue [3]. This dynamic biochemical environment can complicate and often confound the diagnostic evaluation of immature brain tissue on conventional MRI secondary to different signal characteristics compared to those in mature adult brains. However, the consistency of cell proliferation and protein synthesis in the developing brain offers the potential for improved characterization using newer MRI techniques such as MT and APT imaging, which generate contrast through the identification of semisolid macromolecules and proteins (both free proteins and polypeptide chains), respectively. Higher MT and APT values measured in these images reflect relatively higher semisolid macromolecular and protein content. The structural and molecular information provided by MT and APT imaging offers a potentially valuable supplement to conventional MRI examinations in the assessment of brain development.

Our results demonstrated linear, positive correlation between APT and MT values measured in different regions of the brain and patient gestational age, consistent with 
TABLE 1: The measured APT (\%) of frontal lobe deep white matter, basal ganglia, and occipital lobe deep white matter with gestational age (day).

\begin{tabular}{|c|c|c|c|c|}
\hline \multicolumn{5}{|c|}{$\mathrm{APT}(\%)$} \\
\hline Case & Gestational age (day) & Frontal lobe deep white matter & Basal ganglia & Occipital lobe deep white matter \\
\hline 1 & 191 & 0.2865565 & 0.5610845 & 0.2240295 \\
\hline 2 & 196 & 0.1606295 & 0.753212 & 0.531229 \\
\hline 3 & 203 & 0.314636 & 0.417282 & 0.3569482 \\
\hline 4 & 210 & 0.3283785 & 1.04580925 & 0.56413275 \\
\hline 5 & 210 & 0.658413 & 1.0398185 & 0.573494 \\
\hline 6 & 219 & 0.654974 & 1.234728333 & 0.625218 \\
\hline 7 & 219 & 0.247978 & 1.27012 & 0.7158715 \\
\hline 8 & 222 & 0.5333595 & 1.270135 & 0.6364595 \\
\hline 9 & 224 & 0.62417025 & 1.0394645 & 0.87172725 \\
\hline 10 & 229 & 0.6208725 & 0.873164 & 0.6813355 \\
\hline 11 & 233 & 0.581705 & 1.2721025 & 0.65139675 \\
\hline 12 & 237 & 0.388836 & 1.23342 & 0.485775 \\
\hline 13 & 238 & 0.48234873 & 1.357225 & 0.7878892 \\
\hline 14 & 238 & 0.54149115 & 1.554525 & 0.767246 \\
\hline 15 & 240 & 0.1850855 & 1.24202 & 0.58474 \\
\hline 16 & 240 & 0.8021585 & 1.54691 & 0.9852405 \\
\hline 17 & 243 & 0.43556 & 1.07769 & 0.5521545 \\
\hline 18 & 250 & 0.64959525 & 1.403605 & 0.7551365 \\
\hline 19 & 250 & 0.5394515 & 1.433305 & 0.757351 \\
\hline 20 & 252 & 0.7482053 & 1.390218167 & 0.770961333 \\
\hline 21 & 252 & 0.8250045 & 1.48694 & 0.6612495 \\
\hline 22 & 258 & 0.706682 & 1.1035045 & 0.9320605 \\
\hline 23 & 259 & 0.7196395 & 1.15165 & 0.7811165 \\
\hline 24 & 267 & 0.764964 & 1.38085075 & 0.937480875 \\
\hline 25 & 268 & 0.82505 & 1.035838 & 0.896967 \\
\hline 26 & 269 & 0.6293945 & 1.650745 & 1.092922 \\
\hline 27 & 270 & 0.788212 & 1.494025 & 0.8789635 \\
\hline 28 & 275 & 0.9989034 & 1.46188 & 1.085519167 \\
\hline 29 & 277 & 0.9608 & 1.52487 & 1.109135 \\
\hline 30 & 278 & 1.0568445 & 1.445595 & 1.167215 \\
\hline 31 & 279 & 1.02664408 & 1.5563758 & 1.2141181 \\
\hline 32 & 280 & 0.801769 & 1.614184 & 0.97464 \\
\hline 33 & 282 & 1.1601819 & 1.53851 & 1.39712 \\
\hline 34 & 282 & 1.08711 & 1.77139 & 1.28227 \\
\hline 35 & 284 & 1.0008835 & 1.491565 & 1.394685 \\
\hline 36 & 284 & 1.15272 & 1.698 & 1.525505 \\
\hline 37 & 285 & 1.1803 & Null $^{*}$ & Null $^{*}$ \\
\hline 38 & 287 & 1.237795 & 1.796135 & 1.558985 \\
\hline & Mean \pm SD & $0.70 \pm 0.29$ & $1.30 \pm 0.31$ & $0.86 \pm 0.32$ \\
\hline
\end{tabular}

${ }^{*}$ Null means APT values measurement failed in basal ganglia and occipital lobe due to the presence of partial artifacts of case 37.

known physiological changes during CNS development, namely, neuroglial cell proliferation and myelination [37]. The observed correlation between MTR values and patient age in the current study is consistent with those reported in prior investigations $[29,32]$. As showed in Figure 3 , the $R^{2}$ values were relative small compared to the APT findings. This is due to the fact that brain development is a gradual process of myelination and glial cells proliferation. In the brain, MT effect primarily originates from semisolid macromolecular including cholesterol and other lipids [13, 32, 38], which 
TABLE 2: The measured MTR (\%) of frontal lobe deep white matter, basal ganglia, and occipital lobe deep white matter with gestational age (day).

\begin{tabular}{|c|c|c|c|c|}
\hline \multicolumn{5}{|c|}{ MTR (\%) } \\
\hline Case & Gestational age (day) & Frontal lobe deep white matter & Basal ganglia & Occipital lobe deep white matter \\
\hline 1 & 191 & 11.0947 & 16.6731 & 12.1622 \\
\hline 2 & 196 & 12.6331 & 14.9308 & 10.91905 \\
\hline 3 & 203 & 10.52812 & 18.89185 & 11.2131 \\
\hline 4 & 210 & 12.6491 & 19.88995 & 12.0236 \\
\hline 5 & 210 & 10.68809 & 18.02345 & 12.4753 \\
\hline 6 & 219 & 12.7266 & 17.6464 & 13.0213 \\
\hline 7 & 219 & 12.85625 & 17.90015 & 12.4656 \\
\hline 8 & 222 & 10.25065 & 16.3197 & 11.35305 \\
\hline 9 & 224 & 10.37185 & 12.2972 & 12.6507 \\
\hline 10 & 229 & 12.55615 & 13.9513 & 12.126 \\
\hline 11 & 233 & 11.926 & 17.8582 & 13.89715 \\
\hline 12 & 237 & 11.00735 & 16.544 & 12.1135 \\
\hline 13 & 238 & 10.09604 & 17.04255 & 11.88205 \\
\hline 14 & 238 & 10.19335 & 16.16505 & 11.2817 \\
\hline 15 & 240 & 11.05615 & 17.4869 & 12.04665 \\
\hline 16 & 240 & 10.772 & 16.7616 & 12.2732 \\
\hline 17 & 243 & 12.57395 & 19.14885 & 13.6891 \\
\hline 18 & 250 & 13.7127 & 18.099 & 11.9721 \\
\hline 19 & 250 & 10.55205 & 16.5026 & 12.31035 \\
\hline 20 & 252 & 10.27066 & 18.98355 & 13.0489 \\
\hline 21 & 252 & 11.41115 & 20.0092 & 13.8119 \\
\hline 22 & 258 & 13.7253 & 18.84055 & 13.78625 \\
\hline 23 & 259 & 12.4354 & 17.236 & 11.30005 \\
\hline 24 & 267 & 12.3934 & 20.46975 & 13.4312 \\
\hline 25 & 268 & 12.86325 & 15.9051 & 13.58795 \\
\hline 26 & 269 & 11.88875 & 18.43385 & 13.1562 \\
\hline 27 & 270 & 12.3402 & 20.05515 & 13.46845 \\
\hline 28 & 275 & 14.5353 & 23.2817 & 14.7597 \\
\hline 29 & 277 & 12.12065 & 20.6666 & 14.291 \\
\hline 30 & 278 & 14.2746 & 21.6414 & 14.3113 \\
\hline 31 & 279 & 12.5789 & 17.1573 & 14.66385 \\
\hline 32 & 280 & 14.5956 & 20.3918 & 14.3798 \\
\hline 33 & 282 & 12.9527 & 16.2719 & 14.01905 \\
\hline 34 & 282 & 12.06405 & 20.9366 & 13.9 \\
\hline 35 & 284 & 12.79625 & 17.0396 & 11.9296 \\
\hline 36 & 284 & 13.1498 & 22.40645 & 13.1636 \\
\hline 37 & 285 & 13.48875 & 21.6642 & 14.54245 \\
\hline 38 & 287 & 11.1343 & 16.4571 & 12.86825 \\
\hline & Mean \pm SD & $12.09 \pm 1.28$ & $18.16 \pm 2.34$ & $12.90 \pm 1.09$ \\
\hline
\end{tabular}

are important components of myelin sheath. So, MT is often used to evaluate the degree of myelination, while ATP imaging can primarily detect endogenous mobile proteins (such as those dissolved in the cytoplasm) [16], which can reflect the increase of protein content in the process of glial cells proliferation and the formation of myelin sheath. The observation substances of the two imaging methods (MT and APT) are different, and that may be the reason why the correlation between MTR and gestational age is different from that of APT.

APT and MT measurements both showed significant differences between the three brain regions evaluated in this 


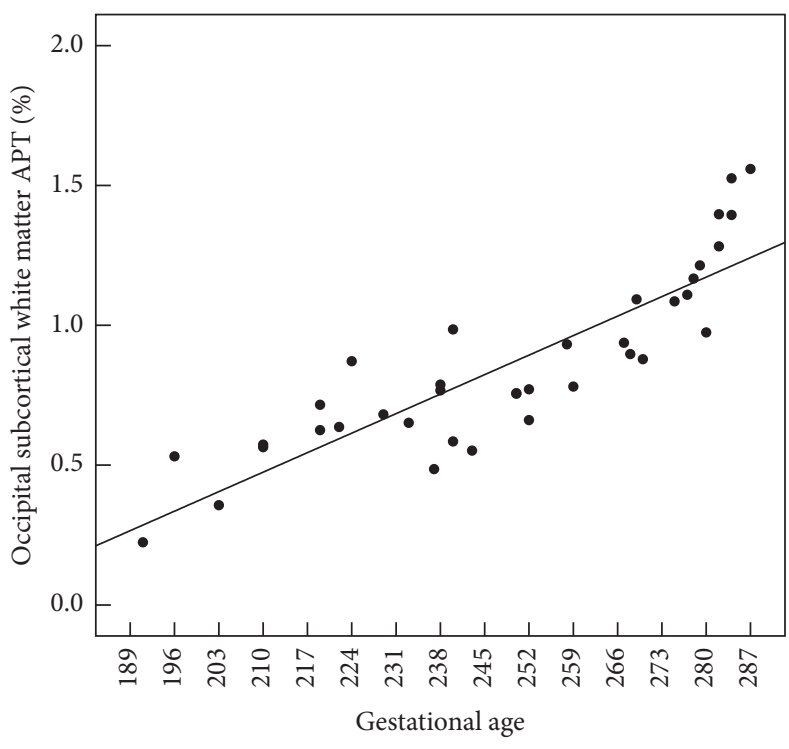

(a)

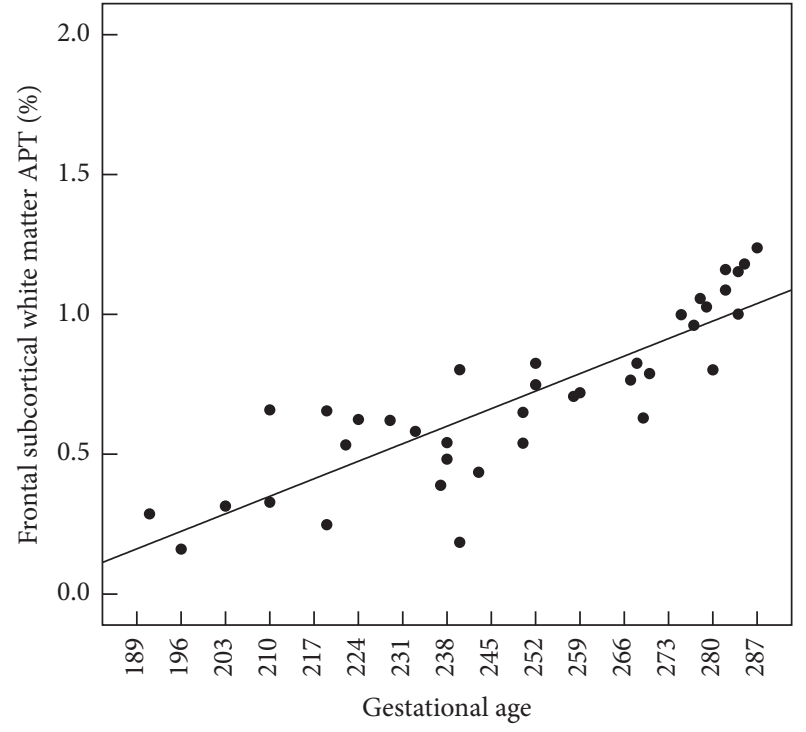

(b)

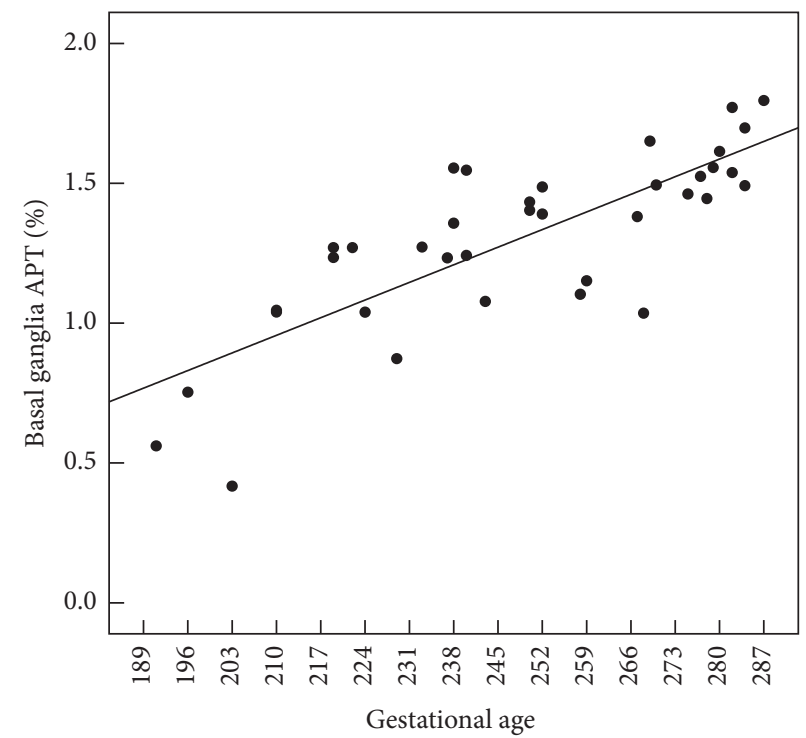

(c)

Figure 2: Correlations between gestational age (days) and APT values in the deep white matter of the occipital lobe (a), deep white matter of the frontal lobe (b), and basal ganglia (c). $R^{2}$ : occipital lobe deep white matter: 0.75 ; frontal lobe deep white matter: 0.73 ; basal ganglia: 0.63 .

study. These regional disparities, however, can be explained by the asymmetric regional progression of neonatal brain maturation with associated underlying differences in tissue composition and inhomogeneity of local internal environments. The basal ganglia, which are rich in perikaryons and dendrites, develop earlier than the frontal and occipital lobes. Moreover, the deep white matter of the occipital and frontal lobes is composed of nerve fibers that are unmyelinated at birth. Furthermore, myelination of white matter occurs later in the frontal lobe than in the occipital lobe. Mean APT and MT values in the current study measured highest in the basal ganglia, followed by the occipital lobe deep white matter, and lowest in the frontal lobe deep white matter. This observed pattern is concordant with the demonstrated directional pattern of myelination [39-43], shown to progress from caudal to rostral, central to peripheral, and dorsal to ventral.

Zhang et al. have also recently performed an imaging study of brain development [18]. They evaluated the use of APT and MT-MRI in the characterization of pediatric brain development and observed that the APT signal in the brain decreased with increasing patient age. Although the ages of the participants in their study ranged from 0 to 16 years of age, no neonates were included in the study population, and only 16 participants were between the ages of 0 and 2 years. Consequently, the observed signal changes characterize a patient population at a later stage of the maturation spectrum 


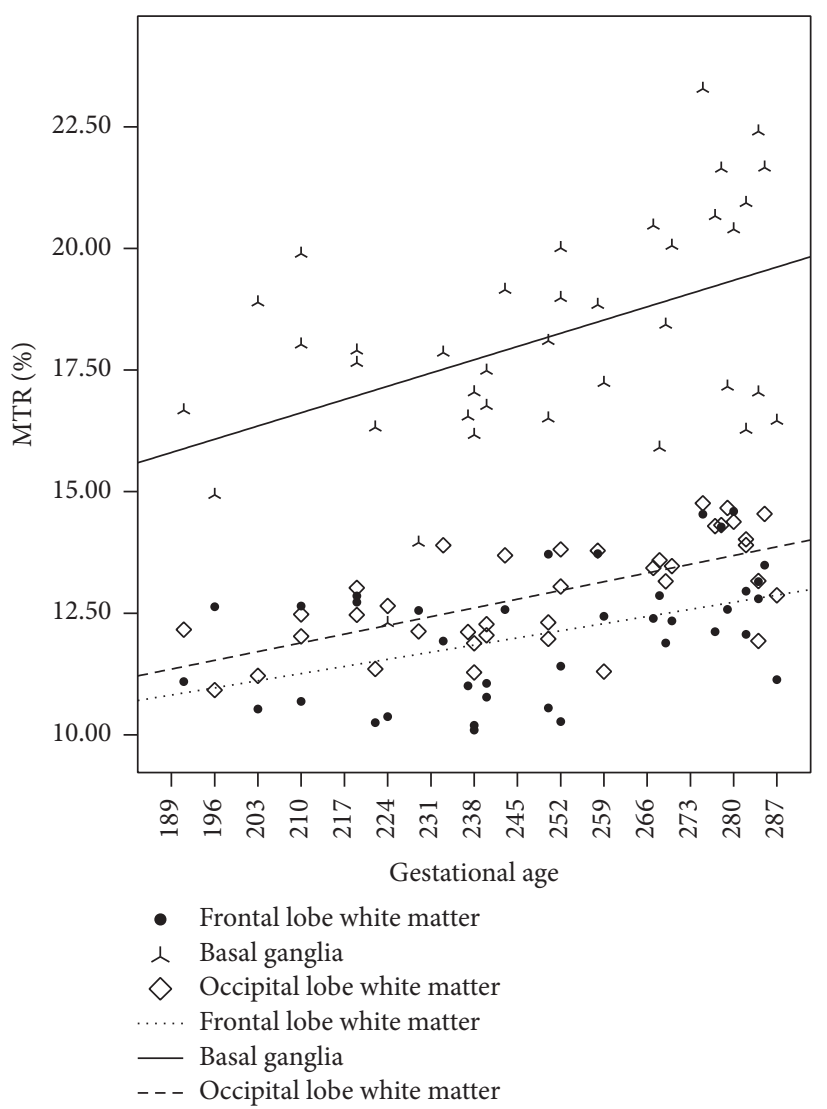

FIGURE 3: Correlations between MTR values in the brain and patient gestational age. The thin dotted line, the solid line, and the bold dotted line reflect MTR measurements in the frontal lobe white matter, basal ganglia, and occipital lobe white matter, respectively. $R^{2}$ values are as follows: occipital lobe: 0.43 , frontal lobe: 0.21 , and basal ganglia: 0.21 .

than that of the current study, with more advanced degrees of myelination. In contrast, our study evaluated APT/MT signal changes within patients between 27 and 41 weeks' gestational age. The inhomogeneous biochemical and physiological patterns of maturation across different age ranges could feasibly manifest similarly different trends of APT signal change over time.

Despite the promising results of our study, several technical and performance issues must be addressed prior to routine clinical application of the APT imaging. For example, issues that limit APT/MT in its current state include eliminating direct water saturation effect, increasing the SNR, and improving image contrast $[44,45]$. Safety concerns also warrant consideration, in regard to the specific absorption rate (SAR) of radiofrequency energy and the trade-off with acceptable scan time, saturation power, and flip angle.

There were several limitations in our study regarding the use of APT/MT imaging for evaluation of neonatal brain development. Firstly, our investigation is limited by the technical limitations of our available APT/MT imaging sequence, which permits only single-slice imaging. Thus, for the axial basal ganglia level selected in this study, other areas and structures were neither shown nor evaluated. Also, our study was limited to some degree by the somewhat small size of the patient population. Theoretically, the small number of patients within each gestational age group could have fostered slight bias in measured correlations between APT/MT signals and different gestational ages. However, we do not expect that the size of the patient population group should have affected the trend of APT/MT measurements across the different gestational ages.

\section{Conclusions}

Neonatal brain development is associated with cell proliferation and increased protein content. APT and MT offer noninvasive means of characterizing this process through the quantification of protein content and myelination. Applied to the neonatal brain, APT and MT imaging offer effective new tools for the characterization of brain maturation that could enhance our understanding of normal and pathologic brain development.

\section{Abbreviations}

MT: Magnetization transfer

APT: Amide proton transfer

MTR: Magnetization transfer ratio

CEST: Chemical exchange saturation transfer. 


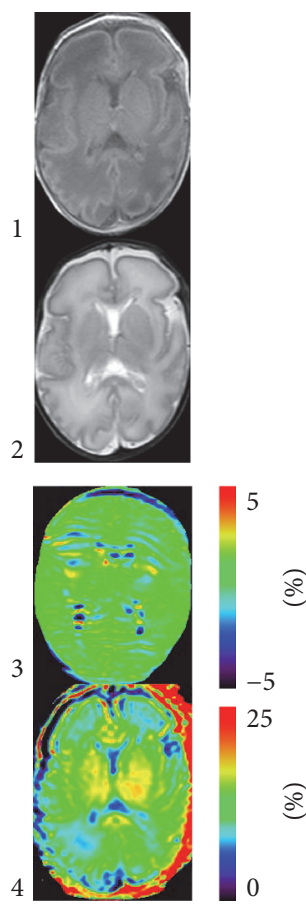

(a)

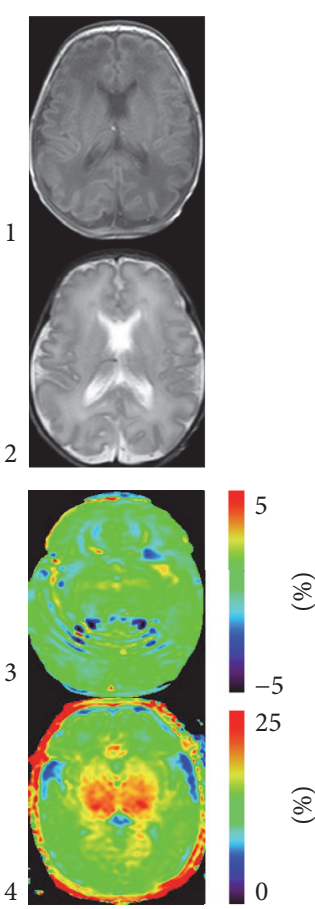

(b)

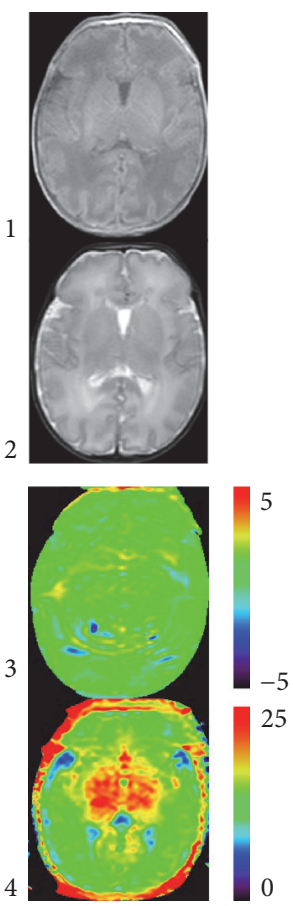

(c)

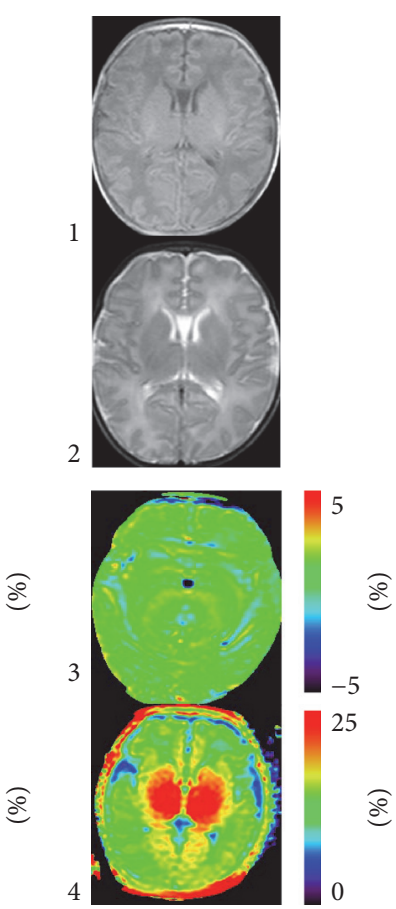

(d)

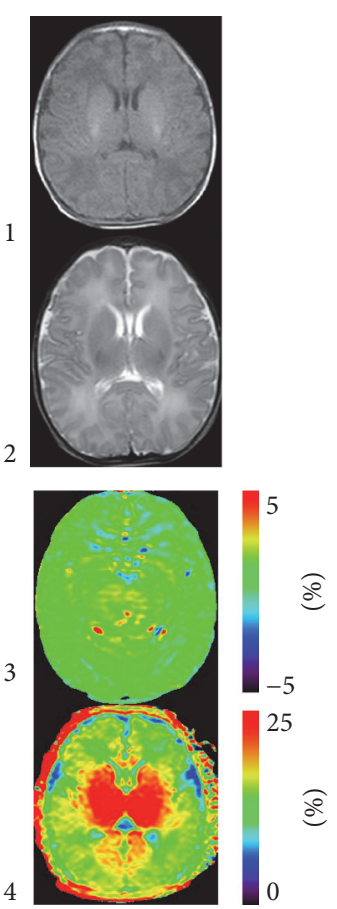

(e)

FIGURE 4: Axial images from examples of neonatal brain MRI at different corrected gestational ages. Columns (a)-(e) represent images from neonates with corrected gestational ages of $28 w, 33 w+2 d, 35 w+5,37 w$, and $40 w+3 d$, respectively. Images from the 4 rows are as follows: row 1 = T1WI; row 2 = T2WI images; row 3 = APT images; and row 4 = MTR images. From Figure 4, we can conclude that, with increased growth associated with age, the T2WI hyperintensity region of deep white matter showes decreased T2 hyperintensity; the formation of myelin in the posterior limb of the internal capsule is revealed as T2WI hypointensity and T1WI hyperintensity. The APT signal appears to gradually increase (signal is somewhat obscured by image contrast). Bright and dark regions (artifacts from cerebrospinal fluid (CSF)) can be seen around bilateral lateral ventricles and sulci. These scattered signals decrease with the increasing gestational age. Because with the myelination and proliferation of glial cells, the water content of brain tissue decreases, the sulci of brain becomes narrow, and the signal interference of CSF decreases. The MT signal is increased with gestational ages.

\section{Competing Interests}

The authors declare that there is no conflict of interests regarding the publication of this paper.

\section{Acknowledgments}

This study was supported by National Natural Science Foundation of China (no. 30570541, 30770632, and 81271631) and the NIH Grant P41 EB015909.

\section{References}

[1] E. Ortiz, J. M. Pasquini, K. Thompson et al., "Effect of manipulation of iron storage, transport, or availability on myelin composition and brain iron content in three different animal models," Journal of Neuroscience Research, vol. 77, no. 5, pp. 681689, 2004.

[2] N. Baumann and D. Pham-Dinh, "Biology of oligodendrocyte and myelin in the mammalian central nervous system," Physiological Reviews, vol. 81, no. 2, pp. 871-927, 2001.

[3] W. Deng and R. D. Poretz, "Oligodendroglia in developmental neurotoxicity," NeuroToxicology, vol. 24, no. 2, pp. 161-178, 2003.
[4] J. H. Gilmore, W. Lin, M. W. Prastawa et al., "Regional gray matter growth, sexual dimorphism, and cerebral asymmetry in the neonatal brain," Journal of Neuroscience, vol. 27, no. 6, pp. 1255-1260, 2007.

[5] W. Gao, W. Lin, Y. Chen et al., "Temporal and spatial development of axonal maturation and myelination of white matter in the developing brain," American Journal of Neuroradiology, vol. 30, no. 2, pp. 290-296, 2009.

[6] P. B. Toft, H. Leth, H. C. Lou, O. Pryds, and O. Henriksen, "Metabolite concentrations in the developing brain estimated with proton MR spectroscopy," Journal of Magnetic Resonance Imaging, vol. 4, no. 5, pp. 674-680, 1994.

[7] J. Rose, R. Vassar, K. Cahill-Rowley et al., "Neonatal physiological correlates of near-term brain development on MRI and DTI in very-low-birth-weight preterm infants," NeuroImage: Clinical, vol. 5, pp. 169-177, 2014.

[8] A. I. Bartha, K. R. L. Yap, S. P. Miller et al., "The normal neonatal brain: MR imaging, diffusion tensor imaging, and 3D MR spectroscopy in healthy term neonates," American Journal of Neuroradiology, vol. 28, no. 6, pp. 1015-1021, 2007.

[9] U. Sonnewald, N. Westergaard, E. Isern et al., "MRS evaluation of brain metabolites in extracts from cell cultures, human tumors and normal tissue from brain: cholesteryl ester, choline 
containing compounds and creatine as markers for development, differentiation and pathology," International Journal of Oncology, vol. 2, no. 4, pp. 545-555, 1993.

[10] N. Girard, C. Fogliarini, A. Viola et al., "MRS of normal and impaired fetal brain development," European Journal of Radiology, vol. 57, no. 2, pp. 217-225, 2006.

[11] M. S. van der Knaap, J. van der Grond, P. C. van Rijen, J. A. J. Faber, J. Valk, and K. Willemse, "Age-dependent changes in localized proton and phosphorus MR spectroscopy of the brain," Radiology, vol. 176, no. 2, pp. 509-515, 1990.

[12] R. M. Henkelman, G. J. Stanisz, and S. J. Graham, "Magnetization transfer in MRI: a review," NMR in Biomedicine, vol. 14, no. 2, pp. 57-64, 2001.

[13] W. Kucharczyk, P. M. Macdonald, G. J. Stanisz, and R. M. Henkelman, "Relaxivity and magnetization transfer of white matter lipids at MR imaging: importance of cerebrosides and pH," Radiology, vol. 192, no. 2, pp. 521-529, 1994.

[14] P. Z. Sun, T. Benner, W. A. Copen, and A. G. Sorensen, "Early experience of translating $\mathrm{pH}$-weighted MRI to image human subjects at 3 Tesla," Stroke, vol. 41, no. 10, pp. S147-S151, 2010.

[15] J. L. Markley, A. Bax, Y. Arata et al., "Recommendations for the presentation of NMR structures of proteins and nucleic acids. IUPAC-IUBMB-IUPAB inter-union task group on the standardization of data bases of protein and nucleic acid structures determined by NMR spectroscopy," European Journal of Biochemistry, vol. 256, no. 1, pp. 1-15, 1998.

[16] J. Zhou, J.-F. Payen, D. A. Wilson, R. J. Traystman, and P. C. M. Van Zijl, "Using the amide proton signals of intracellular proteins and peptides to detect $\mathrm{pH}$ effects in MRI," Nature Medicine, vol. 9, no. 8, pp. 1085-1090, 2003.

[17] R. A. Kauppinen, H. Kokko, and S. R. Williams, "Detection of mobile proteins by proton nuclear magnetic resonance spectroscopy in the guinea pig brain ex vivo and their partial purification," Journal of Neurochemistry, vol. 58, no. 3, pp. 967974, 1992.

[18] H. Zhang, H. Kang, X. Zhao et al., "Amide Proton Transfer (APT) MR imaging and Magnetization Transfer (MT) MR imaging of pediatric brain development," European Radiology, vol. 26, no. 10, pp. 3368-3376, 2016.

[19] O. Togao, A. Hiwatashi, K. Yamashita et al., "Grading diffuse gliomas without intense contrast enhancement by amide proton transfer MR imaging: comparisons with diffusion- and perfusion-weighted imaging," European Radiology, 2016.

[20] J. E. Park, H. S. Kim, K. J. Park, S. J. Kim, J. H. Kim, and S. A. Smith, "Pre-and posttreatment glioma: comparison of amide proton transfer imaging with MR spectroscopy for biomarkers of tumor proliferation," Radiology, vol. 278, no. 2, pp. 514-523, 2016.

[21] J. E. Park, H. S. Kim, K. J. Park, C. G. Choi, and S. J. Kim, "Histogram analysis of amide proton transfer imaging to identify contrast-enhancing low-grade brain tumor that mimics highgrade tumor: increased accuracy of MR perfusion," Radiology, vol. 277, no. 1, pp. 151-161, 2015.

[22] L. Gerigk, B. Schmitt, B. Stieltjes et al., "7 Tesla imaging of cerebral radiation necrosis after arteriovenous malformations treatment using amide proton transfer (APT) imaging," Journal of Magnetic Resonance Imaging, vol. 35, no. 5, pp. 1207-1209, 2012.

[23] A. Sakata, T. Okada, A. Yamamoto et al., "Grading glial tumors with amide proton transfer MR imaging: different analytical approaches," Journal of Neuro-Oncology, vol. 122, no. 2, pp. 339348, 2015.
[24] J. Zhou, J. O. Blakeley, J. Hua et al., "Practical data acquisition method for human brain tumor amide proton transfer (APT) imaging," Magnetic Resonance in Medicine, vol. 60, no. 4, pp. 842-849, 2008.

[25] Z. Wen, S. Hu, F. Huang et al., "MR imaging of high-grade brain tumors using endogenous protein and peptide-based contrast," NeuroImage, vol. 51, no. 2, pp. 616-622, 2010.

[26] S. D. Wolff and R. S. Balaban, "Magnetization transfer contrast (MTC) and tissue water proton relaxation in vivo," Magnetic Resonance in Medicine, vol. 10, no. 1, pp. 135-144, 1989.

[27] R. I. Grossman, J. M. Gomori, K. N. Ramer, F. J. Lexa, and M. D. Schnall, "Magnetization transfer: theory and clinical applications in neuroradiology," Radiographics, vol. 14, no. 2, pp. 279-290, 1994.

[28] S. D. Wolff and R. S. Balaban, "Magnetization transfer imaging: practical aspects and clinical applications," Radiology, vol. 192, no. 3, pp. 593-599, 1994.

[29] V. Engelbrecht, M. Rassek, S. Preiss, C. Wald, and U. Mödder, "Age-dependent changes in magnetization transfer contrast of white matter in the pediatric brain," American Journal of Neuroradiology, vol. 19, no. 10, pp. 1923-1929, 1998.

[30] V. Dousset, R. I. Grossman, K. N. Ramer et al., "Experimental allergic encephalomyelitis and multiple sclerosis: lesion characterization with magnetization transfer imaging," Radiology, vol. 182, no. 2, pp. 483-491, 1992.

[31] J. T. Chen, D. L. Collins, H. L. Atkins et al., "Magnetization transfer ratio evolution with demyelination and remyelination in multiple sclerosis lesions," Annals of Neurology, vol. 63, no. 2, pp. 254-262, 2008.

[32] M. A. Van Buchem, S. C. A. Steens, H. A. Vrooman et al., "Global estimation of myelination in the developing brain on the basis of magnetization transfer imaging: A Preliminary Study," American Journal of Neuroradiology, vol. 22, no. 4, pp. 762-766, 2001.

[33] F. Plum and R. W. Price, "Acid-base balance of cisternal and lumbar cerebrospinal fluid in hospital patients," The New England Journal of Medicine, vol. 289, no. 25, pp. 1346-1351, 1973.

[34] H. Kazemi and D. C. Johnson, "Regulation of cerebrospinal fluid acid-base balance," Physiological Reviews, vol. 66, no. 4, pp. 9531057, 1986.

[35] M. Bradl and H. Lassmann, "Oligodendrocytes: biology and pathology," Acta Neuropathologica, vol. 119, no. 1, pp. 37-53, 2010.

[36] N. Girard, C. Raybaud, and P. du Lac, "MRI study of brain myelination," Journal of Neuroradiology, vol. 18, no. 4, pp. 291307, 1991.

[37] N. Herschkowitz, "Brain development in the fetus, neonate and infant," Biology of the Neonate, vol. 54, no. 1, pp. 1-19, 1988.

[38] A. J. Barkovich, "Concepts of myelin and myelination in neuroradiology," American Journal of Neuroradiology, vol. 21, no. 6, pp. 1099-1109, 2000.

[39] A. J. Barkovich, B. O. Kjos, D. E. Jackson Jr., and D. Norman, "Normal maturation of the neonatal and infant brain: MR imaging at 1.5 T," Radiology, vol. 166, no. 1 I, pp. 173-180, 1988.

[40] K. Oishi, S. Mori, P. K. Donohue et al., "Multi-contrast human neonatal brain atlas: application to normal neonate development analysis," NeuroImage, vol. 56, no. 1, pp. 8-20, 2011.

[41] J. Dobbing and J. Sands, "Quantitative growth and development of human brain," Archives of Disease in Childhood, vol. 48, no. 10, pp. 757-767, 1973. 
[42] K. Ruoss, K. Lövblad, G. Schroth, A. C. Moessinger, and C. Fusch, "Brain development (Sulci and Gyri) as assessed by early postnatal MR imaging in preterm and term newborn infants," Neuropediatrics, vol. 32, no. 2, pp. 69-74, 2001.

[43] C. B. McArdle, C. J. Richardson, D. A. Nicholas, M. Mirfakhraee, C. K. Hayden, and E. G. Amparo, "Developmental features of the neonatal brain: MR imaging. Part I. Gray-white matter differentiation and myelination," Radiology, vol. 162, no. 1, pp. 223-229, 1987.

[44] D. Liu, J. Zhou, R. Xue, Z. Zuo, J. An, and D. J. J. Wang, “Quantitative characterization of nuclear overhauser enhancement and amide proton transfer effects in the human brain at 7 Tesla," Magnetic Resonance in Medicine, vol. 70, no. 4, pp. 1070-1081, 2013.

[45] R. Scheidegger, E. Vinogradov, and D. C. Alsop, "Amide proton transfer imaging with improved robustness to magnetic field inhomogeneity and magnetization transfer asymmetry using saturation with frequency alternating RF irradiation," Magnetic Resonance in Medicine, vol. 66, no. 5, pp. 1275-1285, 2011. 


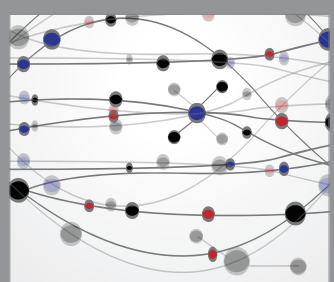

The Scientific World Journal
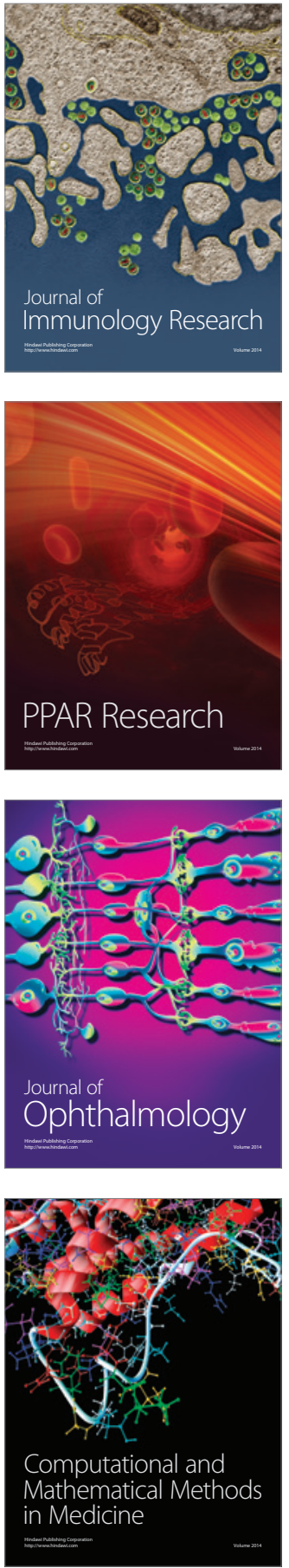

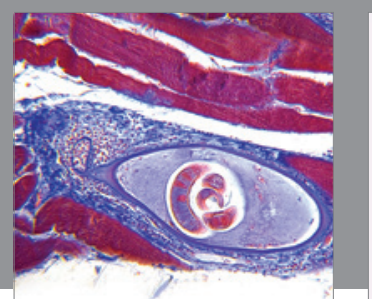

Gastroenterology Research and Practice

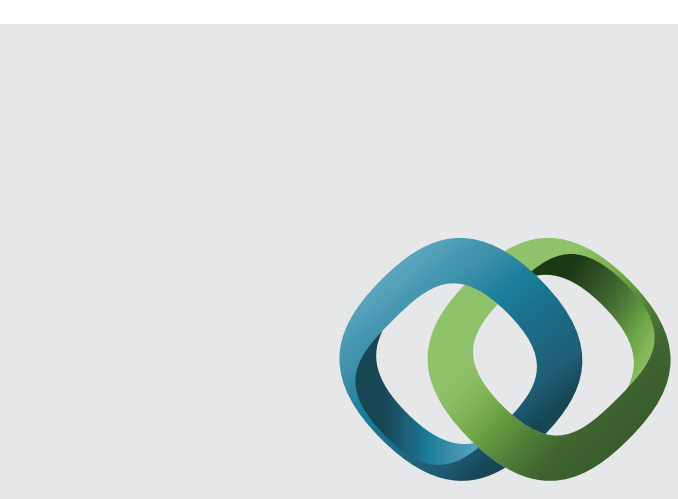

\section{Hindawi}

Submit your manuscripts at

http://www.hindawi.com
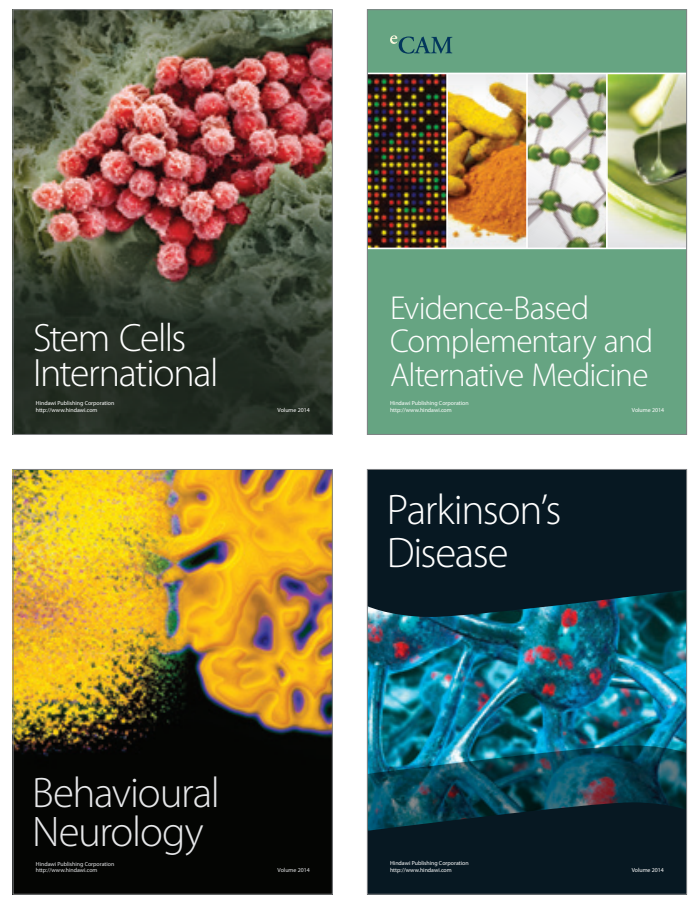
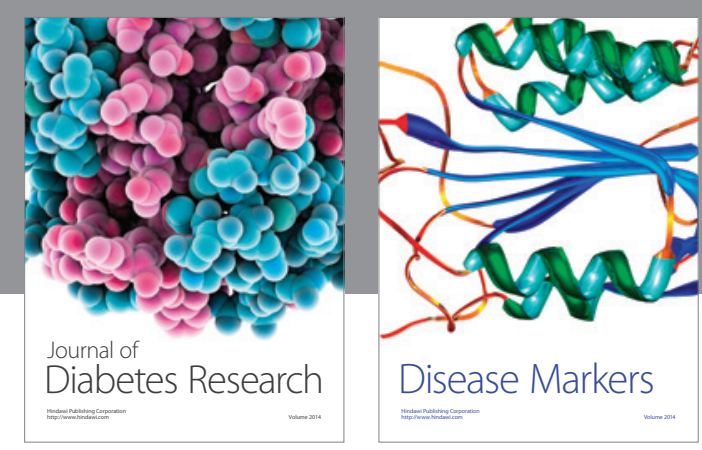

Disease Markers
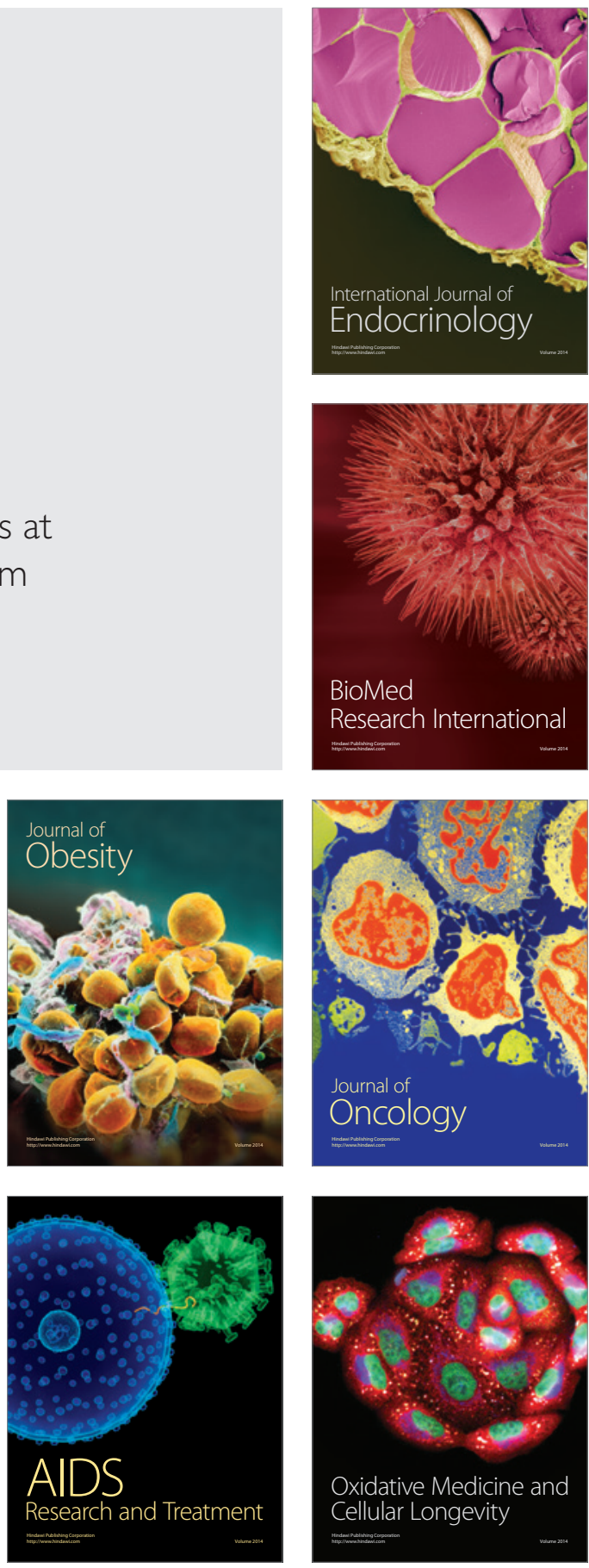